\title{
Nurses and their Caring Behaviours: A Study Summary of the International Caring Behavior Index for Nurses Deduced from Different Voices
}

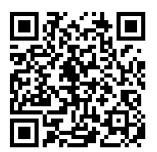

Nio Noveno*

St Paul University Manila, California

*Corresponding author: Nio Noveno, St Paul University Manila, Chula Vista, San Diego, California

Submission: 侮 August 25, 2017; Published: 海 March 13, 2018

\section{Introduction}

Caring has been the cornerstone of nursing since the time of its conception. It has been the legacy that each nurse has been instilled to all those they have interactions with. Many nursing experts have looked into caring as a science of nursing. It has been well studied by many nursing experts. All of these prove that nurses' best asset is to make others feel that they are 'cared for'. However, very few have stated that behaviors that nurses possess [1].

In a qualitative study that I made, it revealed sets of behaviors that resulted from the analyses of the different responses from participants that included nurses themselves, patients and their significant others, and other healthcare professionals [2-4]. Careful analysis of these data has led to the six (6) international caring behavior index.

\section{Perform patient-centric care}

Nurses' goal is the comfort of the patient [5] Focusing on the needs of the patient starts with knowing the patient. This will aid nurses in identifying the unique needs that require more attention. The nurses' role is to find out and meet the patient's immediate need for help (Orlando, n.d.). Orlando's theory describes that nurses' use nursing process in order to discover the true meaning of the patients' behaviors. Patient-centered care requires having a clinical eye which can help identify the salient focus for assessment and plan of care based on the identified problem/needs (fFBRCode_1_ Lanz). The following are caring behavior categories under this theme

\section{Show professional compassion to patients and family}

Compassion is an expression of sympathy. The nurse reveals this through the regular and continuous interaction with the patients and their family members. Most patients want to feel that they are considered as a family member and not just someone who require medical management. Participant iPTS_Code1_DB stated, "Caring is showing one's love and concern for others and always considering their best interest outside oneself. It always aims for the good of the other person." Participant iPTSCode_1_Penalty said, “...wag maramdaman ng patient na patient s'ya kundi parang sa bahay lang, at home ang feeling" (The patient needs to feel that being in the healthcare facility is like being home)." Treating everyone the way you treat your own family (iRNCode_1_Uk) is a way of showing compassion to patients. In addition, knowing how it feels to become a patient and a family who has someone suffering from any health condition (fFRSCode_1_Araneta) makes a professional nurse more compassionate. Being compassionate to others especially to those who are unable to care for themselves (fRY1Code_1_Mg) really matters. However, rather than being considered like a family member by nurses having to be "treated as a patient who is in need of utmost care, patience, and understanding (iPTSCode_1_Sha) is what matters. Nevertheless, professional compassion is essential in caring for patients and their family.

Sympathy can be modulated into other ways of feeling with and for the person in the empathy process, but these sympathyreplacement feelings nevertheless always display some form of motivating concern for the target [6]. Hence, showing compassion should always evolve around the nurses' professional compassion for the patients and family. Otherwise, it can cause nurses to have compassion fatigue [7] if too much sympathy will be felt for patients and family.

\section{Prioritize patient's welfare}

Holistic care involves caring for the patients' overall condition. This would include providing a systematic approach of care that includes physical, psychological and spiritual aspects (fRY2Code_1_ Whyne). Caring for them should focus on the patients' well-being (fjPNCode_1_Vener) and giving everything they need (fJPNCode_1_ Jc) necessary in the caring processes. Furthermore, caring involves listening, acting, empathizing, and fulfilling one's' needs (fRY2Code_1_Amish) [8]. 


\section{Prepare a care plan involves nursing process}

Setting goals for optimum care to achieve optimum health (iRNCode_1_Grandcanyon) as well as "good time management" (iRNCode_1_Uk) are necessary to include in setting goals. The key to a successful nursing care plan are conducting continuous assessment patients (iOHPCode_1_La) and spending more time with them (fFRSCode_1_GraceH, iPTSCode_1_Annecurtis). Furthermore, nurses have more time to assess patients than doctors (iOHPCode_1_Shihder) [9].

\section{Promote comfort}

Physical discomforts and anxieties of patients are some of the many things that nurses need to address. Keeping patients in mild (titrated) sedation using a standard scale to keep them calm and relax (iRNCode_1_Msicu) is an essential intervention among patients especially when they are in deep pain [6-10]. Even pediatric cardiac patients who underwent catheterization should be positioned comfortably (iRNCode_1_Maverick) after the procedure.

\section{Enhance compliance through health education}

Giving them [patients] information about the procedure (fRY2Code_1_Anna) and reassuring them that whatever demand will be taken care of (fRY2Code_1_Mary) are included in the health education process [11]. Involving the significant others by explaining thoroughly the matters involving the patient (Explain ko po nang maayos para maintindihan ng bantay, fFRSCode_1_Abrildz) is part of the health teaching. Hence, conducting health education (fFRSCode_1_Jedz) will eventually improve wrong practices (fFRSCode_1_Macam).

\section{Protect rights and assure safety of patients}

One way of making sure that patients' and significant others are safe in the healthcare facility is to keep them informed in order to meet their expectations hence, lessening their anxieties. Introducing oneself and telling them the names of the ones who will deal with them (fRY2Code_1_Mary) such as the pertinent information (i.e., relating to treatment) of other nurses and their attending physician's name are important. Whenever a medication is being administered explaining the action of drug to the patient before giving it them and providing information on how to take this drug at home during discharge (fFBRCode_1_Jmmac). Nurses also very careful whenever medications are being administered (fRY2Code_1_Kat). Moreover, the health education being given to the patients does not only address their need to understand but, being able to take care of themselves while in the healthcare facility [12-16]. All patients need their own space and time even though they are in the healthcare facility. Maintaining privacy and dignity (iOHPCode_1_Missindia) is another way of protecting their rights as patients. This is also in relation to exhibiting reverence to individualities [17].

\section{Express affinitive demeanor}

Communication is an essential aspect of nursing care. This is essential in making a successful nurse-patient relationship in a way helping others identify their felt difficulties. The close connection of nurses to their patients makes caring more successful. stressed that the nurses' ability to understand own behavior to help others identify perceived difficulties is an important behavior that the nurse should possess. The nurse and patient mutually communicate, establish goals, and take action to attain goals. The way nurses behave during the interaction matters a lot in gaining patients' (and families) participation in the care process. In the same way, the manner in which patients and family respond to this interaction affect nurses' behavior. Patients, their family members, and even other healthcare colleagues have certain uniqueness and peculiarities that make dealing with them more challenging. This makes the nurse-patient relationship more challenging. The following are the behaviors that need to be observed in order to achieve this:

\section{Maintain effective communication}

Verbal and nonverbal techniques (fFBRCode_1_Lanz) such as the use of sign language or paper and pen (fJPNOHP_1_Jc); and using effective touch (iPTS_1_RedNose, iOHP_1_Sanfo) are helpful in maintaining an effective communication. Nurses also greet and ask patients how they feel (iOHPCode_1_Mama) and conduct a systematic extraction of information (iOHPCode_1_Shihder) using a good tone of voice (iPTS_1_AnneCurtis, fFRSCode_1_Araneta) in a courteous (fFBRCode_1_Lanz) and polite (fFBRCode_1_Jmmac) manner. In addition, holding them [patients] and be there for them [patients] when they are unable to care for themselves (fJPNCode_1_ Stephen) are maintaining effective communication [18].

\section{Develop a trusting relationship}

Establishing rapport with patients (fRY2Code_1_Amish, fFRSCode_1_Sinchongco) and even with the patient's family (fRY2Code_1_Mary) is the initial goal of communication. If trust exists between the nurse and the patient cooperation is achieved. According to Participant iRNCode_1_Grandcanyon nurses should be "sensitive...and timely in responding to patients' patients. The participant continues that having "good eye contact; avoiding distractions; showing empathy; and clarifying concerns" are important in gaining patients' trust [18].

\section{Exhibit reverence to individualities}

Nurses are diverse. Each patient is unique. There are participants who were in settings in which culture and religion are predominantly influential in everyone's lives. Moreover, these people also speak a different language than the participants. This affects not only communication but, the caring process and the necessary interventions needed to be done for the patients. This is the sociocultural variable (Neuman, n.d.) which refers to system functions that relate to social and cultural expectations and activities of patients [19].

However, nurses show adaptation to and respect for the country's religion and religious practices; cultural rituals; individual beliefs; and other differences. 


\section{Adaptable to culture and language}

Learning to speak or at least an effort to understand the language is important for nurses to make the care process possible. In countries, such as the KSA and Japan in which language is written and spoken differently than the usual nurses learn to be openminded (fJPNCode_1_Vener) and be flexible (fRY2Code_1_Mary, fRY2Code_1_Kat). Nurses should take time to listen to the patients (fRY2Code_1_Amish) because they [patients] also help (fRY2Code_1 Kat) nurses as much as possible. Patients' expect nurses adjust to them and their situation. Exhibit deference to cultural and religious practices; differences in beliefs; and language barrier Respecting all patients' needs, rights, and beliefs (iOHPCode_1_MissIndia) is important for them. There are certain rituals and practices that nurses must be able to understand as being important for patients. The prayer time, for example, is an important part of daily living for Saudi patients [17-21]. Nurses allow patients to practice their religion fRY2Code_1_Kat) at any given time with precaution on their current health condition and physical capacities. Privacy is also very critical to all patients especially in Japan and KSA where culture is extremely conservative.

In Japan, nurses should not cross the uchi line (fjPNCode_1_ Robin) hence, they should provide different ways of caring considering (fJPNCode_1_Dulay). In the KSA, female nurses should take care of female patients and at most privacy (i.e., covering a woman's face) should be provided (iRNCode_1_Maverick). Showing deference to patients' individualities really matter not to the success of caring but, mostly to the patients themselves [22].

\section{Practice excellent work principles}

Nurses strive hard to fulfill their responsibilities not only to their patients but, also to their profession. They are bound by their working principles and ethical values of the profession [23].

\section{Inspire cheerfulness}

Patients are admitted in a health facility for various reasons whether it's major or minor it surely puts a stall in their normal lives. Despite being tired it is very important that nurses smile (fRY2Code_1_Mary) in front of the patients and even have a sense of humor (fRY2Code_1_Mg) because these helps relieve them of whatever they are currently enduring [23,24]. Despite having irritating patients and heavy workloads nurses still manage to... give the best expression (fJPNCode_1_Fatima). Nurses know how to uplift patients' spirit especially in emotionally-challenging times (iPTSCode_1_Jersey). Conversely, nurses feel gratified realizing that they were able to make a difference to the lives of patients (iPTSCode_1_Maria, fJPNCode_1_Stephen, fJPNCode_1_Robin).

\section{Act as a role model}

By having a positive behavior towards colleagues and patient's nurses are a good role model (iOHPCode_1_MissIndia). For nurses working in the KSA and Japan the way the native people look at and treat them determines their fate. Despite being treated unfairly (fJPNCode_1_Jc) they still work professionally. Some patients in
KSA even curse and treat nurses as slaves (fRY1Code_1_Amish). However, this doesn't stop them from performing their caring roles [25].

\section{Demonstrate a focus on practice}

Nurses follow the written protocols and rules in the proper way (iOHPCode_1_George). However, sometimes some [nurses] will improvise as long as patients will get the benefit from the intervention (fRY1Code_1_Lynnette). Nurses follow the specific institutional policies and professional nursing laws in their respective countries of practice [26].

\section{Dignify profession}

Nurses preserve their dignity by acting professionally toward their colleagues and patients. They don't allow themselves to be slaves or door rugs of others (fRY2Code_1_Mary, fTBKCode_1_ Wire). Nurses assert their rights (fTBKCode_1_Luis) and roles (fTBKCode_1_Jesu) in the team as a care provider. They make sure that the care delivery is always at the priority. Asserting themselves diplomatically especially with doctors whenever they insist on something or asking favors from nurses [26,27]. According to fRY2Code_1_Rheleyn, “Nurses don't want to be treated as slaves then act as professionals. Don't give them the chance to treat you as their slaves..."

\section{Identify boundaries}

Setting limits between what the professional role entails and the kind of personal relationships (iOHPCode_1_La) nurses demonstrate to the patient is important. Clarity (iRNCode_1 Maverick) in this boundary makes an ethical profession. According to them not being able to emotionally detach oneself from the patients would affect future care transactions with other patients.

\section{Demonstrate unwavering patience}

Nurses devote more time to bring efficient, safe, and quality care (fTBKCode_1_Jesu) and in order to provide better care they adapt to the respective institutions' strict policies (fJPNCode_1_ Vener). Nurses' unwavering patience is faced with attending to the demands of patients (fRY2Code_1_Mg) and pressure from colleagues in addition to the expected workload that they need to accomplish. Extended patience will be instrumental in achieving care goals for the patients [27].

\section{Exude wisdom-built presence}

Nurses are expected to be knowledgeable and skillful professionals. They are experts in providing care which is grounded to the foundations learned from their education. The learning's gained as they go through their daily experiences as professionals are building on their wisdom as professional nurses. The way they care is influenced by their experiences and many other significant factors. The function the nurse performs is a complex and creative, as offering unlimited opportunity for the application of the physical, biological, and social sciences and the development of skills based on them (Henderson, 1960). 


\section{Demonstrate clinical competence}

The goal of the healthcare team is ensuring that patients receive the necessary management in order for them to regain the normal lives that they had. To achieve this, nurses play an important role which requires clinical competence. Patients are very critical in the nurses' clinical competence. Nurses need to practice independence which is based in their job description or current scope of practice (iOHPCode_1_Shihder). They need to attend to the patients from assessment to evaluation and assist them from admission to discharge (iRNCode_1_Aussie). Nurses are confident in what they are doing and know when to call for assistance (i.e., referring to the doctors) to provide better management for the patients (iPTSCode_1_Rednose).

\section{Exhibit knowledge of patients and their clinical management}

Nurses are expected to have the fundamental [and advanced] knowledge and skills as they enter the professional practice. They demonstrate competence that are expected from them such as but not limited to giving medications (iOHPCode_1_Missindia); administering therapies or procedures (iOHPCode_1_Shihder); carrying out doctors' orders; and others which exhibit their knowledge of patients and their conditions. Nurses also should exhibit understanding of the procedures done for the patients.

\section{Enhance knowledge and skills}

Enriching their [nurses] knowledge and skills by attending to continuing education seminars and trainings (iRNCode_1_MSICU, iRNCode_1_Kkh) are part of professional growth. There is some internal motivation of wanting to learn (fRY1Code_1_Mg) that drives them to do so. Although sometimes health institutions do not provide for their seminar or training fees (fRY2Code_1_Kat) they still strive to enhance themselves even spending from their own pockets.

\section{Demonstrate professional influence}

Nursing leadership is increasingly gaining popularity in the healthcare industry. This is driven by the fact that nurses' expanding role is acknowledged as significant in patients' care management. A caring leadership is one way to influence their nursing colleagues and even other healthcare team members to effect change. This professional influence is significant in realizing the caring goals for the patients [19-26].

\section{Lead the team}

Teamwork is influenced by leadership (iOHPCode_1_La). Head nurses must be able to show support to their staff in any issues the latter deal with and elevating it to the higher managers (fRY2Code_1_Rheleyn) whenever the situation calls for it. For example, calling the attention of colleagues especially when they treat mothers harshly (iRNCode_1_Maverick) is one way of leading the team. Helping colleagues (iOHPCode_1_La) and making them feel that they are valuable members of the team (iRNCode_1_Kkh) is affecting teamwork. Leadership prevents chaos (fRY1Code_1_ Lynnette) and promotes a good system (fRY1Code_1_Mg).

\section{Safeguard colleagues}

Looking out for one another is vital in working with the other members of the healthcare team. If there is someone who committed [or about to commit] an error nurses call the attention of the person (fRY2Code_1_Mary, fRY2Code_1_Kat). The mistake must be discussed with the colleague and the necessary corrections must be done. Otherwise, if repeated mistakes are observed reporting it to the manager would be a decision to make however, for something that do not cause harm to anyone continues guidance will be given (fRY1Code_1_Cathy).

\section{Assist colleagues}

Working as a team...is vital...in case that the assigned staff is busy with another patient, other staff assists the doctors (fRY2Code_1_Rheleyn). They make sure that they also seek help from others (fRY2Code_1_Amish, fRY2Code_1_Kat). Calling the attention of a trusted colleague (iOHPCode_1_LA) or referring to an authority (fRY2Code_1_Kat, fJPNCode_1_Vener) to do the job is always considered.

\section{Coordinate with other healthcare team members}

Doctors always write orders for patients which requires clarity and understanding by the nurses before implementing the orders (fjPNCode_1_Stephen). In times when patients need financial assistance nurses coordinate with social workers (iPTSCode_1_ Jersey) in order to address the matter. This is important in order to ascertain timely and accurate care delivery [27].

The results of this research reveal that there are important caring behaviors that are threaded among nurses from different areas of nursing in different countries based on the perspective of RN themselves, PTS, and OHP. The findings were congruent with other previous research studies. However, as compared to previous research studies the International Caring Behavior Index for Nurses as Deduced from Different Voices included nurses from different areas of nursing practice, patients with different stable medical conditions, relatives of patients, and other health care providers such as CNA, LVN, medical technologist, and doctors who come from different healthcare facility in different countries.

It is recommended that further research on caring behaviors be done using quantitative analysis in order to strengthen the findings with numerical evidence. Furthermore, it will also be important to utilized the results to come up with a caring behavior tool that will be useful in many areas of nursing. In the actual clinical nursing practice, these behaviors are going to impact HCAHPS (Hospital Consumer Assessment of Healthcare Providers and Systems) scores of healthcare institutions and improve compliance to the Joint Commission accreditation requirements. 
Creative Commons Attribution 4.0 International License

For possible submissions Click Here

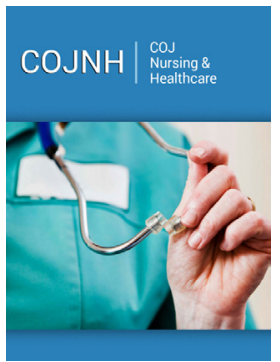

\section{COJ Nursing \& Healthcare}

Benefits of Publishing with us

- High-level peer review and editorial services

- Freely accessible online immediately upon publication

- Authors retain the copyright to their work

- Licensing it under a Creative Commons license

- Visibility through different online platforms 